\section{Zulassung von Fachärzten für Innere Medizin und Kardiologie zum Kolloquium für Computertomografie nach der Vereinbarung zur Strahlendiagnostik und -therapie?}

\section{Einleitung}

Damit ein Arzt Leistungen in der vertragsärztlichen Versorgung erbringen und abrechnen darf, muss er die fachlichen Voraussetzungen nach den Vorschriften des Weiterbildungs-, Qualitätssicherungs- und Vertragsarztrechts sowie nach sonstigen gesetzlichen Vorgaben erfüllen. Dies gilt in der vertragsärztlichen Versorgung insbesondere für Leistungen nach § 135 Abs. 2 SGB V, die wegen der Anforderungen an ihre Ausführung oder wegen der Neuheit des Verfahrens besondere Kenntnisse und Erfahrungen (Fachkundenachweis) erfordern.

Für diese Leistungen können die Kassenärztliche Bundesvereinigung und der GKVSpitzenverband einheitlich entsprechende Voraussetzungen für die Ausführung und Abrechnung dieser Leistungen vereinbaren. Da dies gerade auch im Zusammenhang mit der Erbringung von Leistungen gilt, die eine Anwendung ionisierender Strahlung am Menschen beinhalten, wurde auf dieser Grundlage die Vereinbarung zur Strahlendiagnostik und -therapie ${ }^{1}$ beschlossen, die die Voraussetzungen für die Ausführung und Abrechnung von Leistungen der diagnostischen Radiologie, der Strahlentherapie und der Nuklearmedizin in der vertragsärztlichen Versorgung regelt.

Nach § 2 der Vereinbarung zur Strahlendiagnostik und -therapie ist die Ausführung und Abrechnung von Leistungen der diagnostischen Radiologie, der Strahlentherapie und Nuklearmedizin im Rahmen der vertragsärztlichen Versorgung durch die an der vertragsärztlichen Versorgung teilnehmenden Ärzte insbesondere erst nach Erteilung der Genehmigung durch die Kassenärztliche Vereinigung zulässig. Die Genehmigung ist zu erteilen, wenn der Arzt die Voraussetzungen der fachlichen Befähigung (Abschnitt B) und der apparativen

1 Abrufbar unter: https://www.kbv.de/media/ sp/Strahlendiagnostik.pdf.
Ausstattung (Abschnitt C und Anlage II) der Vereinbarung erfüllt.

Diese Voraussetzungen gelten nach der Vereinbarung auch für den Bereich der Computertomografie (im Folgenden auch: $\mathrm{CT}$ ). Nach $§ 7$ Abs. 1 der Vereinbarung gilt die fachliche Qualifikation durch die Vorlage ausreichender Zeugnisse als nachgewiesen, soweit die Weiterbildungsordnung für eine Weiterbildung in der Computertomografie den Erwerb eingehender Kenntnisse, Erfahrungen und Fertigkeiten im jeweiligen Bereich (Ganzkörper, Kopf) vorschreibt. Diese spezifischen Anforderungen werden gegenwärtig ausschließlich in den Weiterbildungsinhalten des Fachgebiets Radiologie aufgeführt (vgl. Muster-Weiterbildungsordnung 2018 der Bundesärztekammer, Gebiet Radiologie). Die Durchführung der Computertomografie und die Vermittlung von Kenntnissen im Strahlenschutz sind nach der Muster-Weiterbildungsordnung und den Weiterbildungsordnungen der Landesärztekammern dagegen nicht Gegenstand der Weiterbildung zum Facharzt für Innere Medizin und Kardiologie (Kardiologen).

Anderen Ärzten ist allerdings die Erbringung computertomografischer Leistungen in der GKV unter den Voraussetzungen des $\S 7$ Abs. 2 und 3 der Vereinbarung prinzipiell möglich. Dies gilt insbesondere für Ärzte anderer Fachrichtungen, die auf der Grundlage früherer Weiterbildungsordnungen die vorgegebenen Voraussetzungen erfüllen. Soweit keine Weiterbildung nach Abs. 1 stattgefunden hat, kann ein Arzt nach Abs. 2 durch die Vorlage ausreichender Zeugnisse nachweisen, dass er während der genannten Zeiten in der Computertomografie unter der Leitung eines zur Weiterbildung ermächtigten (befugten) Arztes tätig gewesen ist.

Ärzte, die ihre fachliche Qualifikation nach $\S 7$ Abs. 2 der Vereinbarung erworben haben, müssen diese allerdings nach Abs. 3 in einem Kolloquium nach $\S 17$ Abs. 1 S. 2 der Vereinbarung nachweisen.

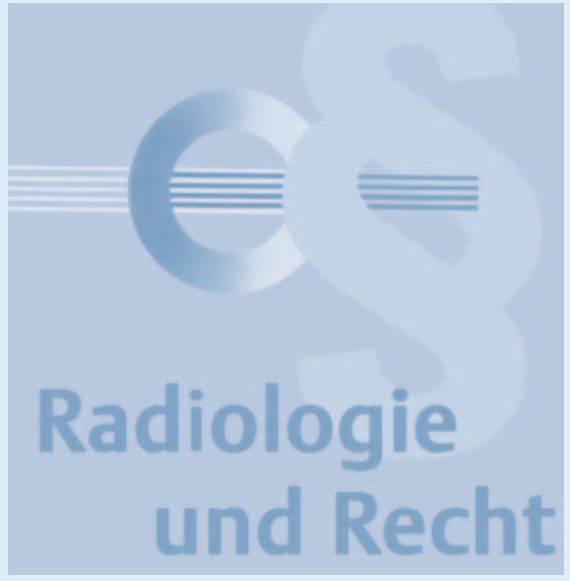

In $§ 7$ Abs. 2 i. V. m. § 17 Abs. 1 S. 2 der Vereinbarung ist geregelt, dass für den Fall, dass der antragstellende Arzt im Vergleich zu § 7 Abs. 1 der Vereinbarung eine abweichende, aber gleichwertige Befähigung im Bereich der Computertomografie nachweist, die Genehmigung für die Ausführung und Abrechnung der computertomografischen Leistungen von der erfolgreichen Teilnahme an einem Kolloquium abhängig ist.

Die Regelung in $\S 17$ Abs. 1 S. 2, wonach die zuständige Kassenärztliche Vereinigung die Abrechnungsgenehmigung auch dann von der erfolgreichen Teilnahme an einem Kolloquium abhängig machen kann, wenn „der antragstellende Arzt im Vergleich zu dieser Vereinbarung eine abweichende, aber gleichwertige Befähigung nachweist", bereitet in der Praxis der Prüfungskommissionen der Kassenärztlichen Vereinigung jedoch regelmäßig Auslegungsschwierigkeiten.

Am Beispiel eines Facharztes für Innere Medizin und Kardiologie soll daher nachfolgend erläutert werden, ob und ggf. unter welchen Voraussetzungen für Fachärzte anderer Fachgebiete die Möglichkeit besteht, an einem Kolloquium teilzunehmen, um zukünftig computertomografische Leistungen in der vertragsärztlichen Versorgung erbringen und abrechnen zu können. Dabei werden die verschiedenen rechtlichen Aspekte beleuchtet, die für eine Einladung eines Facharztes für Innere Medizin und Kardiologie zu einem Kolloquium, das dem Nachweis seiner fachlichen Befähigung im Bereich der Computertomografie dienen soll, von Bedeutung sind. 


\section{Die fachliche Befähigung des Arztes nach der Vereinbarung zur Strahlendiagnostik und -therapie}

Zur Erteilung der erforderlichen Genehmigung zur Erbringung und Abrechnung computertomografischer Leistungen in der GKV gemäß § 2 der Vereinbarung zur Strahlendiagnostik und -therapie vom 10.02.1993 in der geänderten, am 01.01.2020 in Kraft getretenen Fassung vom 09.12.2019 (im Folgenden lediglich: Vereinbarung) ${ }^{2}$ durch die Kassenärztliche Vereinigung ist neben der Gewährleistung einer spezifisch definierten apparativen Ausstattung auch die Erfüllung fachlicher Voraussetzungen in Abhängigkeit zur jeweiligen Anwendungs- oder Untersuchungsmethode durch den Arzt erforderlich (§3 der Vereinbarung).

Für die fachliche Befähigung des Arztes schreibt der hierfür wesentliche $\S 4$ der Vereinbarung grundsätzlich vor:

„Den Anforderungen an die fachliche Befähigung für die Ausführung und Abrechnung von Leistungen der diagnostischen Radiologie ${ }^{3}$ wird entsprochen, wenn der Arzt

1. die für den Strahlenschutz erforderliche Fachkunde nach §47 Strahlenschutzverordnung (StrISchV) nachweist ${ }^{4}$ und

2. eine fachliche Qualifikation gemäß den in den $\S \S 5$ bis 8 genannten Anforderungen erworben hat."

2 Die Vereinbarung ist nach $\S 1$ Abs. 3 BMV-Ä als Anlage 3 Bestandteil des Bundesmantelvertrages-Ärzte.

3 Wie die Systematik der Vereinbarung zeigt, zählen die in den $\S \S 5$ bis 8 der Vereinbarung genannten Utersuchungsmethoden zu den Leistungen der diagnostischen Radiologie, hierzu zählt mithin auch die Computertomografie nach $\S 7$. Dies sind jene Leistungen, die auch der $\S 4 \mathrm{Nr} .2$ der Vereinbarung, der nach seinem Wortlaut eben Abrechnungsvoraussetzungen für "Leistungen der diagnostischen Radiologie" festlegt, in Bezug nimmt. Darüber hinaus zeigt auch der $\S 14$ Abs. 1 S. 1 der Vereinbarung, dass zwischen Leistungen der diagnostischen Radiologie, der Strahlentherapie und der Nuklearmedizin zu unterscheiden ist.

4 Hierzu Näheres in diesem Beitrag unter dem Abschnitt „Strahlenschutzrechtliche Vorgaben nach dem StrlSchG und der StrlSchV“.

\section{Erwerb der fachlichen Befähigung nach Weiterbildungsrecht ( $\$ 7$ Abs. 1)}

Die Anforderungen, die gemäß § 4 Nr. 2 der Vereinbarung an die fachliche Qualifikation des Arztes speziell für den Bereich der Computertomografie gestellt werden, finden sich im $§ 7$ der Vereinbarung.

$\S 7$ Abs. 1 der Vereinbarung hat den Wortlaut (sämtliche Hervorhebungen im Dokument durch die Verfasser):

\section{„(1) Soweit die Weiterbildungsordnung für} eine Weiterbildung in der Computertomographie den Erwerb eingehender Kenntnisse, Erfahrungen und Fertigkeiten in der jeweiligen computertomographischen Diagnostik (Ganzkörper, Kopf) vorschreibt, gilt die fachliche Qualifikation durch die Vorlage ausreichender Zeugnisse als nachgewiesen."

Insofern verweist die Vorschrift auf die Bestimmungen der (jeweils gültigen) Weiterbildungsordnung, die für den antragstellenden Arzt maßgeblich ist. Da die Gebietsdefinition die Grenzen der Ausübung der fachärztlichen Tätigkeit bestimmt ${ }^{5}$ (vgl. §2 Abs. 2 S. 2 der Muster-Weiterbildungsordnung 2018), richtet sich die Genehmigungsfähigkeit in fachlicher Hinsicht grundsätzlich danach, ob die Erbringung der jeweiligen Leistung Gegenstand der Weiterbildung des diese Leistung erbringenden (Fach-) Arztes ist.

Die entsprechend hinreichenden „Kenntnisse, Erfahrungen und Fertigkeiten" im Bereich der CT-Leistungen in dem spezifischen Umfang nach $\S 7$ Abs. 1 der Vereinbarung (nämlich „Ganzkörper, Kopf“ betreffend) vermittelt auch nach den Vorgaben der im Jahr 2018 neugefassten Muster-Weiterbildungsordnung im Ergebnis nach wie vor ausschließlich die Weiterbildung im

5 Zur Maßgeblichkeit der Fachgebietsgrenzen für die Erbringung und Abrechnung von Leistungen ebenso z. B. BSG, Urteil vom 04.05.2016, Az.: B 6 KA 13/15 R, SozR 4-2500 $\S 135 \mathrm{Nr} 25$ = juris; Urteil vom 30.10.2019, Az.: B 6 KA 22/19 B, RegNr 33581 (BSGIntern) $=$ juris.
Fachgebiet Radiologie. ${ }^{6}$ Somit erfüllt nur die Facharztgruppe der Fachärzte für Radiologie die Genehmigungsvoraussetzung der hinreichenden fachlichen Befähigung nach $\S 7$ Abs. 1 der Vereinbarung. Grundsätzlich können demnach Fachärzte für Radiologie mit Blick auf ihre in der Weiterbildung gewonnene fachliche Qualifikation die Genehmigung erhalten, CT-Leistungen im Bereich der vertragsärztlichen Versorgung selbst zu erbringen und abzurechnen.

Ein Facharzt für Innere Medizin und Kardiologie erwirbt diese fachliche Qualifikation hingegen nicht schon durch seine ärztliche Weiterbildung, da die Weiterbildungsinhalte für den Facharzt für Innere Medizin und Kardiologie keine vergleichbaren Methoden- und Handlungskompetenzen wie für das Gebiet der Radiologie vorsehen. ${ }^{7}$

\section{Nachweis der fachlichen Befähi- gung durch Vorlage ausreichender Zeugnisse ( $§ 7$ Abs. 2)}

Wie bereits oben dargelegt, hat unter bestimmten Voraussetzungen jedoch - als Ausnahme vom zuvor gezeigten Grundsatz - auch ein Arzt eines anderen Fachgebiets die Möglichkeit, die Genehmigung zur Ausführung und Abrechnung von Leistungen im Bereich der Computertomografie zu erhalten. Hierzu muss er jedoch nach $\S 7$ Abs. 2 der Vereinbarung durch die Vorlage ausreichender Zeugnisse nachweisen, dass er während der genannten Zeiten unter der Leitung eines zur Weiterbildung ermächtigten Arztes im Bereich der Computertomografie tätig gewesen ist. Zur Feststellung der fachlichen Befähigung des Arztes ist nach $\S 7$ Abs. 3 i. V.m. §17 Abs. 2 der Vereinbarung ein Kolloquium durchzuführen, in dem die Ärzte den Erwerb ihrer Qualifikation nach § 7 Abs. 2 nachzuweisen haben. Für die Durchführung des Kollo-

6 Die nach der neuen Muster-Weiterbildungsordnung der Bundesärztekammer von 2018 erforderliche Kognitive und Methodenkompetenz und Handlungskompetenz zur „Indikation, Durchführung und Befunderstellung von Untersuchungen aller Körperregionen mit Röntgenstrahlung einschließlich CT“ ist nur in den Weiterbildungsinhalten für den Facharzt für Radiologie enthalten.

7 Hierzu Näheres in diesem Beitrag unter dem Abschnitt „Weiterbildungsrechtliche Aspekte: Fachgebietsgrenze (nähere Erläuterungen)“. 
quiums sind zwei unterschiedliche Konstellationen maßgeblich.

$\S 17$ Abs. 1 der Vereinbarung schreibt hierzu Folgendes vor (sämtliche Anmerkungen in eckigen Klammern im Dokument durch die Verfasser):

"(1) ${ }^{1}$ Bestehen trotz der Berechtigung zum Führen einer Gebiets- bzw. Teilgebietsbezeichnung oder der vorgelegten Zeugnisse begründete Zweifel, dass die in Abschnitt B dieser Vereinbarung festgelegten Anforderungen an die fachlichen Befähigungen erfüllt sind [Situation 1], so kann die Kassenärztliche Vereinigung die Erteilung der Genehmigung für die Ausführung und Abrechnung der beantragten Leistungen von der erfolgreichen Teilnahme an einem Kolloquium abhängig machen. ${ }^{2}$ Das gleiche gilt, wenn der antragstellende Arzt im Vergleich zu dieser Vereinbarung eine abweichende, aber gleichwertige Befähigung nachweist [Situation 2]."

Vor dem Hintergrund einer von einem Arzt begehrten Genehmigung zur Erbringung und Abrechnung von Leistungen im Bereich der Computertomografie ist für die weitere Betrachtung allein die zweite Alternative der geregelten Situationen von Interesse.

Im Rahmen dieses Kolloquiums, also eines solchen zum Zwecke des Nachweises einer vom Arzt erlangten „gleichwertigen Befähigung " im Bereich der Computertomografie, kann Bezugspunkt des Begriffs der "gleichwertigen Befähigung" aus regelungssystematischen Gesichtspunkten nur der bereits genannte § 7 Abs. 2 der Vereinbarung sein. Dieser lautet:

"(2) Soweit eine Weiterbildung nach Abs. 1 nicht stattgefunden hat, hat der Antragsteller durch die Vorlage ausreichender Zeugnisse nachzuweisen, dass er während der genannten Zeiten unter der Leitung eines zur Weiterbildung ermächtigten Arztes tätig gewesen ist: a) Für Untersuchungen des Ganzkörpers auch einschl. Kopf und des Spinalkanals

1. eine mindestens 30 monatige ganztägige Tätigkeit in der radiologischen einschl. neuroradiologischen Diagnostik und

2. eine mindestens 10 monatige ganztägige Tätigkeit in der Computertomographie. b) Für Untersuchungen des Kopfes und des Spinalkanals

1. eine mindestens 18 monatige ganztägige Tätigkeit in der radiologischen einschl. neuroradiologischen Diagnostik und

2. eine mindestens 4monatige ganztägige Tätigkeit in der Computertomographie insbesondere des Kopfes und des Spinalkanals“.

Insofern steht denjenigen Facharztgruppen, die ihre fachliche Befähigung im Bereich der Computertomografie nicht bereits durch die Weiterbildung zum Facharzt erworben haben, nach $\S 7$ Abs. 2 der Vereinbarung die Möglichkeit offen, ihre diesbezügliche Befähigung auf anderem Weg nachzuweisen. Dies geschieht durch die Wahrnehmung der verschiedenen Tätigkeiten im Bereich der CT-Leistungen für die Dauer der genannten Zeiträume unter Leitung eines zur Weiterbildung hinreichend qualifizierten und befugten Arztes.

Die durch diese praktischen Tätigkeiten im ersten Schritt erworbene, anderweitige (aber gleichwertige) Befähigung muss durch die Ärzte in einem zweiten Schritt mittels der Teilnahme an einem Kolloquium nachgewiesen werden (§7 Abs. 3 S. 1 i. V. m. § 17 Abs. 2 lit. b) der Vereinbarung). Um die Genehmigung zur Ausführung und Abrechnung von CT-Leistungen als solche der diagnostischen Radiologie zu erhalten, ist die Teilnahme an diesem Kolloquium für die nicht hinreichend weitergebildeten Facharztgruppen - also letztlich Nicht-Radiologen - demzufolge eine unabdingbare Voraussetzung.

Aus den soeben gemachten Ausführungen ergibt sich jedoch eine wesentliche Unterscheidung mit Auswirkungen auf die Zulassungsvoraussetzungen zum Kolloquium. Denn es ist zwischen dem Erwerb der fachlichen Befähigung auf der einen Seite und dem Nachweis dieser Befähigung auf der anderen Seite zu trennen. Auch wenn der Nachweis über die fachliche Befähigung erst mit der Teilnahme am Kolloquium erfolgt, hat die Kommission bereits im Vorfeld der Einladung des Arztes zum Kolloquium zu prüfen, ob bei ihm die Voraussetzungen erfüllt sind, die der $\S 7$ Abs. 2 der Vereinbarung an den Erwerb einer gleichwertigen fachlichen Befähigung stellt.
In der Beispielsfallkonstellation eines Facharztes für Innere Medizin und Kardiologie, der die Abrechnungsgenehmigung für CT-Leistungen beantragt (hat), ist noch einmal festzuhalten, dass er seine fachliche Qualifikation bzgl. der Erbringung computertomografischer Leistungen nicht bereits aufgrund seiner (fach-) ärztlichen Weiterbildung auf dem Fachgebiet der Inneren Medizin und Kardiologie erworben haben kann. Er kann die fachliche Befähigung jedoch grundsätzlich erwerben, indem er in einem konkret vorgegebenen zeitlichen Umfang unter der Leitung eines anderen Arztes mit Weiterbildungsbefugnis die vorgeschriebenen Tätigkeiten ausgeführt hat und die dadurch erlangte fachliche Befähigung in einem Kolloquium unter Beweis stellt ( $§ 7$ Abs. 2 der Vereinbarung). Damit der antragsstellende Arzt zum Kolloquium zugelassen werden kann, muss er zuvor nachweisen, dass er die notwendigen Tätigkeiten im Bereich der Computertomografie für die jeweils vorgeschriebene Dauer unter fachlich qualifizierter Aufsicht ausgeführt hat. Wird er eingeladen, obwohl er diesen Nachweis nicht erbracht hat, ist diese Einladung in unzulässiger Weise ausgesprochen worden.

Eine Einladung eines Facharztes für Innere Medizin und Kardiologie zur Teilnahme an einem derartigen Kolloquium ist in Ergänzung zu den bisherigen Ausführungen auch an den Vorgaben anderer Regelungsregime, die von den einschlägigen Vorschriften der Vereinbarung auch ausdrücklich in Bezug genommen werden, zu messen. Diese sollen im Folgenden näher behandelt werden.

\section{Weiterbildungsrechtliche} Aspekte: Fachgebietsgrenze (nähere Erläuterungen)

\section{Muster-Weiterbildungsordnung von 2003}

Geltung besitzt für den einzelnen Arzt ausschließlich die jeweils geltende Fassung der Weiterbildungsordnung der für ihn zuständigen (Landes-) Ärztekammer. Die MusterWeiterbildungsordnung der Bundesärztekammer stellt dabei lediglich eine Orientierung bzw. Empfehlung für die jeweiligen Ärztekammern dar. 
Mit Blick auf die hier relevanten Inhalte der Weiterbildung zum Facharzt für Innere Medizin und Kardiologie ist festzustellen, dass die derzeit (noch) geltenden Fassungen der Weiterbildungsordnungen aller Ärztekammern letztlich auf der Muster-Weiterbildungsordnung der Bundesärztekammer aus dem Jahr 2003 in der Fassung vom 23.10.2015 ${ }^{8}$ beruhen; dies gilt in gleicher Weise auch für die jeweils dazugehörigen Richtlinien zu den Inhalten der Weiterbildung, die erkennbar die (Muster-) Richtlinien der Bundesärztekammer aus dem Jahr 2003 in der Fassung vom 18.02.2011 ${ }^{9}$ zur Grundlage haben.

Ein dem radiologischen Leistungsbereich grundsätzlich zuzuordnender Gegenstand der Facharztweiterbildung ist nach der Muster-Weiterbildungsordnung 2003 dort unter Punkt 13.6 - wohl lediglich die dort genannte

„Durchleuchtung, Aufnahmetechnik und Beurteilung von Röntgenbefunden bei Angiokardiographien und Koronarangiographien“.

Die Erbringung von CT-Leistungen ist dagegen nicht ausdrücklich als Gegenstand der Weiterbildung zum Facharzt für Innere Medizin und Kardiologie aufgeführt; dies gilt auch nicht - was grundsätzlich denkbar wäre - für ausschließlich kardiale CT-Leistungen.

Damit gibt es im Regelungsbereich der Muster-Weiterbildungsordnung 2003 im Ergebnis keine Hinweise darauf, dass Fachärzte für Innere Medizin und Kardiologie eingehende Kenntnisse, Erfahrungen und Fertigkeiten in der jeweiligen computertomografischen Diagnostik („Ganzkörper, Kopf“) erworben haben, wie es der $\S 7$ Abs. 1 der Vereinbarung als Genehmigungsvoraussetzung vorsieht.

\section{Muster-Weiterbildungsordnung von 2018}

Die Bundesärztekammer hat am 16.11.2018 eine neue Muster-Weiterbildunasordnuna ${ }^{10}$

8 Abrufbar unter: https://www.bundesaerzte kammer.de/aerzte/aus-weiter-fortbildung/ weiterbildung/muster-weiterbildungsord nung/.

9 Ebd.

10 Ebd. beschlossen. Diese unterscheidet bei der Darstellung der Weiterbildungsinhalte stringent zwischen „Kognitiven und Methodenkompetenzen (Kenntnissen)“ und „Handlungskompetenzen (Erfahrungen und Fertigkeiten)“. Die (Muster-) Richtlinien zu den Weiterbildungsinhalten entfallen nun ganz; die Weiterbildungsordnung selbst enthält sämtliche Weiterbildungsinhalte sowie Richtzahlen für mindestens durchzuführende Anwendungs- oder Untersuchungsmethoden des jeweiligen Fachgebiets. Als Gegenstand der Weiterbildung zum Facharzt für Innere Medizin und Kardiologie findet sich lediglich die

„Indikationsstellung und Befundinterpretation von Röntgen-Thorax-Untersuchungen“.

Diese unter der Kategorie der Handlungskompetenzen geführte Fähigkeit vermittelt allein jedoch ersichtlich nicht die eingehenden Kenntnisse, wie sie der $\S 7$ Abs. 1 der Vereinbarung vorschreibt.

Ergänzend sei angemerkt, dass im Regelungszusammenhang der Muster-Weiterbildungsordnung 2018 der Begriff der „Indikationsstellung“ von dem Begriff der „rechtfertigenden Indikation “ (Legaldefinition in §8 83 Abs. 3 S. 1 StrlSchG) zu unterscheiden ist; die Stellung der rechtfertigenden Indikation findet sich in dieser Weiterbildungsordnung als Handlungskompetenz ausschließlich in den Inhalten der Weiterbildung zum Facharzt für Radiologie (Radiologen). Daraus ergibt sich, dass eine ggf. infrage stehende Befähigung im Zusammenhang mit der Stellung einer rechtfertigenden Indikation von dem Begriff der „Indikationsstellung“, wie er im obigen Zitat verwendet wird, nicht erfasst ist und die Stellung der rechtfertigenden Indikation damit nicht zu den Gegenständen der Weiterbildung zum Facharzt für Innere Medizin und Kardiologie gehört.

Im Ergebnis wird man auch nach der neuen Muster-Weiterbildungsordnung 2018 der Bundesärztekammer nicht davon ausgehen können, dass eine Facharztweiterbildung zum Facharzt für Innere Medizin und Kardiologie dem Arzt hinreichende Kenntnisse und Fertigkeiten im Bereich der Durchführung der Computertomografie verschafft, um seine fachliche Qualifikation schon durch Vorlage des Facharztzeugnisses im Zusammenhang mit dem Erhalt der Ab- rechnungsgenehmigung für solche Leistungen zu belegen.

Derzeit ist allerdings noch in keinem Zuständigkeitsbereich einer der im Bundesgebiet bestehenden (Landes-) Ärztekammern eine Weiterbildungsordnung auf der Grundlage der Regelungen der Muster-Weiterbildungsordnung 2018 in Kraft getreten. Bei einigen (Landes-) Ärztekammern wurde jedoch eine neue Weiterbildungsordnung bereits beschlossen, die am 01.07.2020 in Kraft treten wird ${ }^{11}$ bzw. ein Inkrafttreten zu diesem Zeitpunkt zumindest beabsichtigt ist ${ }^{12}$.

\section{Strahlenschutzrechtliche Vorgaben nach dem StrlSchG und der StrlSchV}

Des Weiteren sind als grundlegende Voraussetzung für die Anwendung computertomografischer Verfahren strahlenschutzrechtliche Vorgaben einzuhalten. Dies gilt insbesondere auch vor dem Hintergrund der Genehmigungsvoraussetzung, dass der antragstellende Arzt den Nachweis der erforderlichen Fachkunde im Strahlenschutz (gemäß §47 StrlSchV) erbringen muss. Eindeutig gibt dies $\S 4$ Nr. 1 der Vereinbarung vor.

Das Recht des Strahlenschutzes ist mit dem Gesetz zur Neuordnung des Rechts zum Schutz vor der schädlichen Wirkung ionisierender Strahlung vom 27.06.2017 13 und der Verordnung zur weiteren Modernisierung des Strahlenschutzrechts vom 29.11.2018 ${ }^{14}$, vor allem in Umsetzung der Richtlinie 2013/59/Euratom des Rates vom 05.12.2013, auf neue rechtliche Grundlagen gestellt worden.

Aufgrund der vorgenannten Verordnung sind am 31.12.2018 die bis zu diesem Zeitpunkt geltende Röntgenverordnung ${ }^{15}$ (RöV) vom

11 Z. B. LÄK Hessen, Internet-Quelle: https:// www.laekh.de/aerzte/weiterbildung.

12 ÄK Sachsen-Anhalt, Internet-Quelle: https:// www.aeksa.de/www/website/PublicNavigati on/arzt/weiterbildung/k3qzjsip/.

13 BGBI.IS. 1966.

14 BGBI.IS. 2034.

15 Langbezeichnung: Verordnung über den Schutz vor Schäden durch Röntgenstrahlen. 
30.04.2003 ${ }^{16}$ in der zuletzt geänderten Fassung vom 11.12.2014 ${ }^{17}$ und die (alte) Strahlenschutzverordnung ${ }^{18}$ vom 20.07.2001 ${ }^{19}$ in der zuletzt geänderten Fassung vom 27.01.201720 (StrISchV [2001]) außer Kraft getreten. Die in diesen beiden früheren Verordnungen enthaltenen Regelungen finden sich nun zum einen im Strahlenschutzgesetz ${ }^{21}$ (StrlSchG) vom 27.06.201722 - in der inzwischen zuletzt geänderten Fassung vom 12.12.2019 23 - und zum anderen in der aufgrund dieses Gesetzes erlassenen (neuen) Strahlenschutzverordnung (StrlSchV) vom 29.11.201824, die gleichzeitig mit dem Außerkrafttreten der alten Regelungen zum vorgenannten Zeitpunkt in ihren wesentlichen Teilen in Kraft getreten sind.

Die Voraussetzungen der Anwendung ionisierender Strahlung oder radioaktiver Stoffe am Menschen, d. h. im Falle der Computertomografie der technischen Durchführung von Untersuchungen und deren anschließende Befundung (vgl. §5 Abs. 3 Nr. 1 StrlSchG), sind von dem Grad der Befähigung der diese Anwendung durchführenden Person abhängig. Der § 145 Abs. 1 StrlSchV25 bestimmt grundsätzlich, dass Ärzte oder Zahnärzte (wenigstens) 2 Voraussetzungen erfüllen müssen, um ionisierende Strahlung am Menschen anwenden zu dürfen:

1. Zum einen müssen sie approbiert oder ihnen muss wenigstens die vorübergehende Ausübung des ärztlichen oder zahnärztlichen Berufs erlaubt sein,

2. zum anderen müssen diese Personen entweder über die erforderliche Fachkunde im Strahlenschutz (Nr. 1) oder lediglich über die für die Anwendung radioaktiver Stoffe und ionisierender Strahlung erforderlichen Kenntnisse im Strahlenschutz in ihrem speziellen $\mathrm{Ar}$ -

16 BGBI.I S. 604.

17 BGBI. I 2010.

18 Langbezeichnung: Verordnung über den Schutz vor Schäden durch ionisierende Strahlen. BGBI.| S. 1714, 2002 I 1459. BGBI.I S. 114, 1222, 1676. Sesetz zum Schutz vor der schädlichen Wirkung ionisierender Strahlung. BGBI.I S. 1966.

23 BGBI.I S. 2510.

24 BGBI.I S. 2036.

25 Vorher: § 24 Abs. 1 RöV, vgl. Begründung zu $\S 145$ Abs. 1 StrlSchV, BT-Drs. 423/18, S. 452. beitsgebiet verfügen, wobei Letzteres nur unter der einschränkenden Bedingung zulässig ist, dass sie unter ständiger Aufsicht und Verantwortung einer Person, die die genannte Fachkunde im Strahlenschutz besitzt, tätig sind (Nr. 2).

Im Zusammenhang mit der Genehmigung zur Ausführung und Abrechnung von Leistungen ist allerdings nur die Berechtigung nach der Nummer 1 des $§ 145$ Abs. 1 StrISchV relevant, vgl. §4 Nr. 1 der Vereinbarung (Zitat s. oben). Denn die Abrechnungsgenehmigung für Leistungen im radiologischen Bereich kann nach dieser Vorschrift ausschließlich der Arzt erhalten, der die erforderliche Fachkunde im Strahlenschutz nach §47 StrlSchV besitzt.

Neben der technischen Durchführung der Anwendung hat auch die Stellung der sogenannten rechtfertigenden Indikation im Vorfeld der Anwendung diese erforderliche Fachkunde im Strahlenschutz seitens des Arztes als Voraussetzung. Es handelt sich bei der rechtfertigenden Indikation um die Entscheidung des Arztes darüber, so die gesetzliche Definition in $\S 83$ Abs. 3 S. 1 StrISchG ${ }^{26}$, dass und auf welche Weise die jeweilige Anwendung durchzuführen ist.

Vor dem Hintergrund einer Zulassung eines Facharztes für Innere Medizin und Kardiologie zu einem Kolloquium zum Zweck der Erlangung einer Abrechnungsgenehmigung für CT-Leistungen in der GKV ist damit festzustellen, dass der Arzt als Bestandteil seiner fachlichen Befähigung neben der fachlichen Qualifikation auch die erforderliche Fachkunde im Strahlenschutz nachweisen muss. Diese Fachkunde ist - wie schon die Trennung der Nummern 1 und 2 des $\S 4$ der Vereinbarung beweist - von der oben in diesem Beitrag dargestellten fachlichen Qualifikation zur Durchführung von CT-Leistungen zu trennen und im Übrigen auch von der ärztlichen Ausbildung unabhängig ${ }^{27}$, weshalb von ihrem gesonderten Nachweis nicht abgesehen werden kann.

26 Vorher entsprechend: §2 Nr. 10 RöV, § 3 Nr. 17 StrlSchV (2001), vgl. Gesetzesbegründung zu §83 Abs. 1 S. 1 StrlSchG, BT-Drs. 18/ 11241, S. 334

27 Vgl. Schmatz/Nöthlichs, Kommentar zur StrlSchV und RöV (Loseblattsammlung), $\S 18 a$ RöV, Stand: 4. EL 2003.
Der Erwerb der erforderlichen Fachkunde im Strahlenschutz richtet sich im Ausgangspunkt nach $\S 74$ Abs. 1 StrlSchG, in dem es heißt:

„(1) Die erforderliche Fachkunde im Strahlenschutz wird in der Regel durch eine für das jeweilige Anwendungsgebiet geeignete Ausbildung, durch praktische Erfahrung und durch die erfolgreiche Teilnahme an von der zuständigen Stelle anerkannten Kursen erworben. “

Die aufgrund der Verordnungsermächtigung des $\S 74$ Abs. 3 und 4 StrlSchG ergangene Vorschrift des $§ 47$ Abs. 1 StrlSchV28 trifft hierzu die nähere Regelung, dass die zuständige Stelle den Erwerb der erforderlichen Fachkunde im Strahlenschutz prüft und bescheinigt (dort S. 1). Hierzu sind Nachweise über die Erwerbsvoraussetzungen zu erbringen (S. 2). Die notwendige erfolgreiche Teilnahme an anerkannten Kursen darf, so wird näher bestimmt, nicht länger als 5 Jahre zurückliegen (S. 3).

Die zu erwerbende praktische Erfahrung richtet sich in Bezug auf ihre Dauer, die Art und den Umfang nach der Ausbildung des Arztes und dem jeweiligen Anwendungsgebiet ( $\$ 47$ Abs. 2 S. 3 StrlSchV). Eine erfolgreiche Teilnahme an einem anerkannten Kurs ist bei erfolgreicher Absolvierung der sich an den Kurs anschließenden Abschlussprüfung anzunehmen ( $\$ 47$ Abs. 3 S. 4 StrlSchV).

Es zeigt sich, dass in der Strahlenschutzverordnung hinreichend konkrete Vorgaben für die Inhalte der Kurse oder für den Erwerb der praktischen Erfahrung, etwa Mindestzahlen spezifischer durchzuführender Untersuchungen etc., fehlen. Zu dem Strahlenschutzgesetz oder der Strahlenschutzverordnung ergangene Verwaltungsvorschriften, die die zuständigen Behörden bei der Auslegung der demnach in der Vorschrift des $\S 74$ StrlSchG bzw. auch der in der Vorschrift des § 47 StrlSchV enthaltenen unbestimmten Rechtsbegriffe leiten sollen, existieren (bislang) nicht.

Zu der Vorgängervorschrift des § 74 Abs. 1 StrlSchG (vgl. §18a Abs. 1 RöV) wurde am

28 Vorher entsprechend: § 18a Abs. 1 RöV, § 30 Abs. 1 StrlSchV (2001), vgl. Begründung zu $\S 47$ Abs. 1 StrlSchV, BT-Drs. 423/18, S. 375. 
22.12.2005 die Richtlinie Fachkunde und Kenntnisse im Strahlenschutz bei dem Betrieb von Röntgeneinrichtungen in der Medizin oder Zahnmedizin ${ }^{29}$ erlassen, die am 27.06.2012 geändert ${ }^{30}$ und am 28.11.2012 korrigiert worden ist ${ }^{31}$ (im Folgenden: Fachkunde-Richtlinie). Diese enthält nähere Hinweise für die Durchführung der Bestimmungen zum Erwerb der Fachkunde und behält auch nach der beschriebenen Neuregelung des Strahlenschutzrechts ihre Gültigkeit. Zum einen ist eine neue Verwaltungsvorschrift (noch) nicht ergangen. Zum anderen weist die Gesetzesbegründung zu $\S 74$ Abs. 1 StrISchG explizit darauf hin, dass die Elemente der Fachkunde, die in dieser Norm enthalten sind, denen des §18a Abs. 1 S. 1 der RöV nachgebildet sind und ihnen somit entsprechen. ${ }^{32}$ Auslegungsschwierigkeiten drohen also auch bei einer Anwendung der zur alten Rechtslage ergangenen FachkundeRichtlinie auf die neuen Vorschriften nicht zu entstehen. Schließlich geht die Begründung zum §47 Abs. 1 StrlSchV sogar ausdrücklich davon aus, dass die konkrete Ausgestaltung der Anforderungen an den Nachweis der praktischen Erfahrung wie bisher auf Richtlinienebene erfolgt und setzt damit die Fortgeltung auch der Fachkunde-Richtlinie offenbar voraus. ${ }^{33}$

So fordert die Fachkunde-Richtlinie (dort Punkt 4.1.3) für den Nachweis der Fachkunde bei nahezu gleichem Regelungsinhalt wie $§ 47$ Abs. 1 StrlSchV:

„Die berufliche Ausbildung ist durch Zeugnisse, die praktische Erfahrung durch Nachweise der Sachkunde unter Angabe von Zeiträumen und durchgeführten Untersuchungen oder Behandlungen und die erfolgreiche Kursteil-

29 RdSchr. d. BMU v. 22.12.2005 - RS II 1 11 603/01.1, GMBI. 2006, Nr. 22, S. 414.

31 RdSchr. d. BMU v. 28.11.2012 - RS II 4 $11603 / 01$, GMBI. 2012, Nr. 61, S. 1204.

32 Vgl. Gesetzesbegründung zu § 74 Abs. 1 StrlSchG, BT-Drs. 18/11241, S. 320.

33 Vgl. Begründung zu §47 Abs. 2 StrlSchV, BTDrs. 423/18, S. 376; ebenso gilt hiernach die Richtlinie Strahlenschutz in der Medizin vom 17.10.2011 (GMBI. 2011, S. 867) in der geänderten Fassung vom 11.07.2014 (GMBI. 2014, S. 1020) zum bisherigen $\S 30$ Abs. 1 StrISchV (2001) weiter. nahme durch Bescheinigungen zu belegen. Die erfolgreiche Teilnahme am letzten Kurs darf nicht länger als 5 Jahre zurückliegen. [...]“

Sie legt über die Vorschriften des Strahlenschutzgesetzes oder der Strahlenschutzverordnung hinaus in konkreter Weise insbesondere die Dauer und Inhalte der jeweiligen Kurse, die Anzahl der spezifischen durchgeführten Untersuchungen und die Zeiträume der Tätigkeit auf dem jeweiligen Untersuchungs- bzw. Anwendungsgebiet fest.

Der Erwerb der Fachkunde ist aber auch ohne das eben beschriebene Prozedere dadurch möglich, dass die Person eine Berufsausbildung absolviert hat, die nach Feststellung der zuständigen Behörde die für das jeweilige Anwendungsgebiet erforderliche Fachkunde vermittelt ( $\$ 47$ Abs. 5 S. 1 StrISchV). Fachärzte für Innere Medizin und Kardiologie verfügen jedoch regelmäßig nicht über die strahlenschutzrechtliche Qualifikation zur eigenständigen Durchführung von Computertomografien. Allenfalls die nach früheren Weiterbildungsordnungen ausgebildeten Fachärzte für Innere Medizin mit dem sog. Teilgebiet Kardiologie nach den Weiterbildungsordnungen von 1989 bzw. 1993 können in Ausnahmefällen über eine entsprechende Qualifikation verfügen.

Im Ergebnis gilt also, dass in der zugrunde gelegten Beispielkonstellation der Facharzt für Innere Medizin und Kardiologie im Vorfeld des Kolloquiums neben dem Nachweis der fachlichen Qualifikation auch den (gesonderten) Nachweis über die erforderliche Fachkunde im Strahlenschutz zu erbringen hat. Andernfalls darf eine Einladung zu dem Kolloquium nicht erfolgen.

\section{Fazit}

Ein Facharzt für Innere Medizin und Kardiologie, der eine Genehmigung zur Ausführung und Abrechnung von CT-Leistungen in der vertragsärztlichen Versorgung beantragt, muss seine anderweitig erlangte, aber gleichwertige Befähigung in diesem Bereich nach § 7 Abs. 2 der Vereinbarung vor der Entscheidung der Kassenärztlichen Vereinigung über eine Einladung zu einem Kolloquium nach § 7 Abs. 3 der Vereinbarung nachweisen. Dies gilt ebenso für die davon grundsätzlich zu trennende erforderliche Fachkunde im Strahlenschutz.

Dieses Ergebnis resultiert insbesondere daraus, dass die Weiterbildung zum Facharzt für Innere Medizin und Kardiologie nach den derzeit geltenden Weiterbildungsordnungen der (Landes-) Ärztekammern nicht eine den Genehmigungsvoraussetzungen genügende fachliche Qualifikation vermittelt.

Die Neufassung der Muster-Weiterbildungsordnung der Bundesärztekammer im Jahr 2018 und die Ende desselben Jahres in Kraft getretene Neuregelung des Strahlenschutzrechts führen nicht dazu, dass an die Erteilung einer Genehmigung zur Ausführung und Abrechnung von CT-Leistungen in der vertragsärztlichen Versorgung an Fachärzte für Innere Medizin und Kardiologie im Vergleich zur alten Rechtslage andere, gar vereinfachte, Anforderungen zu stellen sind.

Prof. Dr. Peter Wigge

Rechtsanwalt

Fachanwalt für Medizinrecht

Philip Steuwer

Rechtsanwalt

Rechtsanwälte Wigge

Scharnhorststraße 40

48151 Münster

Telefon: (0251) 53 595-0

Telefax: (0251) 53 595-99

E-Mail: kanzlei@ra-wigge.de

www.ra-wigge.de 